\title{
Avant l'écriture, à côté du texte : listes et genèse littéraire
}

\section{Monica Zanardo}

\section{(2) OpenEdition}

1 Journals

Édition électronique

URL : https://journals.openedition.org/genesis/3413

DOI : 10.4000/genesis.3413

ISSN : 2268-1590

Éditeur :

Presses universitaires de Paris Sorbonne (PUPS), Société internationale de génétique artistique littéraire et scientifique (SIGALES)

Édition imprimée

Date de publication : 15 décembre 2018

Pagination : 35-52

ISBN : 979-10-231-06282

ISSN : 1167-5101

\section{Référence électronique}

Monica Zanardo, "Avant l'écriture, à côté du texte : listes et genèse littéraire », Genesis [En ligne], 47 |

2018, mis en ligne le 15 décembre 2019, consulté le 12 janvier 2023. URL : http://

journals.openedition.org/genesis/3413; DOI : https://doi.org/10.4000/genesis.3413 


\title{
Avant l'écriture, à côté du texte : listes et genèse littéraire
}

\author{
Monica Zanardo
}

$\Lambda^{p}$

première vue, rien ne ressemble plus à une liste qu'une autre liste : vocables empilés en colonne ou juxtaposés, litanies énumératives, répertoires, catalogues... Vouloir faire une liste de listes, n'est-ce pas redoubler de monotonie ? Et pourtant, si on considère la fonction qui leur est attribuée par les écrivains dans le cours de la genèse, on perçoit une réelle diversité. C'est le large éventail des listes génétiques, celles que les écrivains dressent au cours de la rédaction d'un ouvrage, que cette contribution se propose d'ouvrir : nous nous pencherons particulièrement sur les listes qui ne figurent pas en tant que telles dans l'œuvre publiée, bien qu'elles en aient nourri la genèse à de multiples degrés. Notre analyse cherche essentiellement à identifier et à comprendre ce qui subsiste de ce type de listes génétiques à l'autre bout du parcours, dans le texte publié.

Sans prétendre à l'exhaustivité, nous réfléchirons, à l'aide de quelques exemples, aux multiples manières par lesquelles les auteurs incorporent les listes au récit, afin de proposer un classement fonctionnel des listes selon leur emploi. Cette exploration des usages des listes ne repose pas sur une description de leurs qualités, mais vise à montrer comment des listes homologues par leur contenu ou par leur structure peuvent être exploitées dans des directions distinctes, voire opposées, ou encore, comment des listes de forme et de contenu analogues peuvent avoir des usages différents d'un scripteur à l'autre au sein d'un même dossier, d'une liste à l'autre. Les différents emplois de ce dispositif reposeraient donc sur la méthode des écrivains, donnant vie à un classement dont les catégories sont poreuses et se réorganisent dans le mouvement général de l'écriture.

Les usages de ces listes, et leur rôle dans la genèse, reposent sur la structure bivalente de la liste, toujours en équilibre entre évocation et détermination, entre souci d'exhaustivité et nécessité de synthèse, entre ouverture et fermeture : ce sont précisément les transformations progressives des listes, notamment lorsqu'elles sont appelées à disparaître au fil de l'écriture, qui font, aux yeux d'un généticien, leur intérêt. Cette contribution portera sur le rôle des listes dans l'avant-texte, afin d'explorer leur fonction génétique et de retracer leurs parcours d'exploitation.

Si une liste est toujours travaillée par une tension entre la singularité des items dont elle se compose et la globalité de la structure qui les englobe ${ }^{1}$ (autrement dit, entre les éléments et l'ensemble), on peut dire que lorsque le scripteur rédige une liste, sa structure est travaillée par accumulation et ajout progressif d'items, alors que ces derniers, par leur caractère

$1 . \ll[\ldots]$ the list is simultaneously the sum of its parts and the individual parts themselves. By accretion, the separate units cohere to fulfill some function as a combined whole, and by discontinuity the individuality of each unit is maintained as a particular instance, a particular attribute, a particular object or person. Like the conjunction and, the list joins and separates at the same time» (Robert E. Belknap, The List. The Uses and Pleasures of Cataloguing, New Haven, Yale University Press, 2004, p. 15).

Listes expansives et listes contractiles 
unitaire, sont le résultat d'un effort de synthèse et de réduction : les liens logiques manquent et la syntaxe est réduite au minimum. En revanche, lorsque cette même structure de la liste est utilisée comme point d'appui pour la rédaction d'un texte, elle semble emprunter deux chemins différents : aux listes expansives, qui tendent à l'accroissement, s'opposeraient, par leur fonctionnement, les listes contractiles qui sont progressivement resserrées. Alors que les premières sont le support d'ajouts successifs, les secondes font l'objet d'un processus de sélection : les écrivains éliminent peu à peu des items pour n'en retenir que quelques-uns, voire qu'un seul. Dans le cas des listes expansives, la tension entre la singularité des items et la structure qui les englobe est destinée à basculer vers un équilibre d'ensemble alors que, dans les listes contractiles, ce sont les items qui prendront le dessus, dans leur singularité. Dans le premier cas, les items seront progressivement déployés, et la structure de la liste sera noyée dans l'agencement du récit; dans le second cas, le processus d'accumulation qui a donné naissance à la liste empruntera le chemin opposé, à travers un travail d'élimination et de rétrécissement.

À côté des listes expansives et contractiles, on trouve aussi dans certains dossiers génétiques des listes de régie, qui sont utilisées pour structurer et organiser la matière (aussi bien en amont qu'en aval de la phase rédactionnelle), et des listes d'appui, dont les usages possibles sont très variés : elles peuvent servir, selon le cas, d'aide-mémoire, de témoignage ou, encore, de réservoir d'informations. À l'instar des expansives et des contractiles, les listes de régie et les listes d'appui sont destinées, le plus souvent, à ne pas laisser de traces visibles dans le texte final.

Distincts par leur fonctionnement, les différents usages des listes n'interviennent pas aux mêmes moments de la genèse d'une œuvre. Le plus souvent, les listes expansives précèdent (et parfois accompagnent) la phase rédactionnelle : elles relèvent donc d'une écriture «à programme». Une liste expansive que l'on retrouve fréquemment dans les brouillons d'écrivains est la liste thématique; parmi les manuscrits du poète Andrea Zanzotto (1921-2011), par exemple, Francesco Venturi a repéré et étudié une intéressante liste thématique qui prépare les sujets du recueil Le Galatée au bois ${ }^{2}$ : le poète y mélange des mots («lucioles », «pelleteuse»), des clichés du discours (par exemple «trous de mémoire») et des références culturelles («les indécidables de Derrida») qui portent aussi bien sur des poèmes déjà achevés que sur quelques poèmes qui seront rédigés par la suite, pour être intégrés au Galatée au bois. La disposition de ces éléments hétérogènes sous forme de liste montre les thèmes-clés qui vont nourrir et structurer le recueil.

Bien que ces listes témoignent souvent des premiers moments de la création littéraire, au fil de l'écriture l'écrivain tend à dépasser la liste thématique : une fois sa fonction accomplie, elle est déclassée au rang de document préparatoire et, dans nombre de cas, détruite. Ce dépassement caractérise, d'une manière encore plus évidente, le travail sur les listes contractiles. Les listes de titres, par exemple, qui figurent dans de nombreux dossiers de genèse, l'illustrent parfaitement : leurs auteurs, après avoir répertorié les différents titres possibles, commencent ensuite à les trier, jusqu'au choix définitif (fig. 1).

2. Andrea Zanzotto, Le Galatée au bois, traduit de l'italien par Philippe di Meo, Nantes, Arcane 17, 1986 (en langue originale Il Galateo in bosco, Milan, Mondadori, 1978). Pour l'étude détaillée de cette liste, voir Francesco Venturi, Genesi e storia della «trilogia» di Andrea Zanzotto, Pise, ETS, 2016, notamment les p. 75-89. 


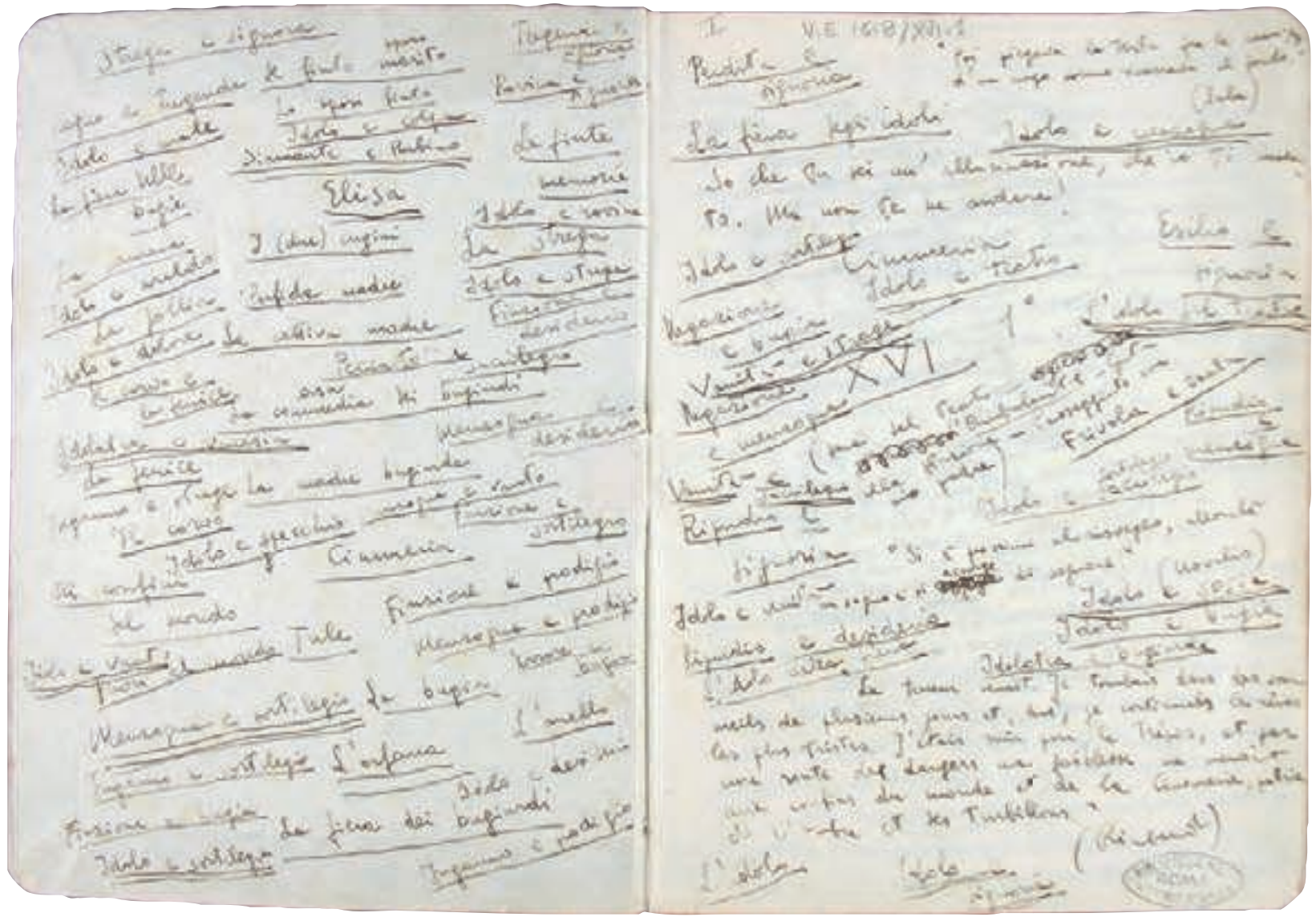

Fig. 1 : Elsa Morante, avant-texte de Menzogna e sortilegio (Mensonge et sortilège), cahier XVI. Rome, Biblioteca Nazionale Centrale, V.E. 1619/XVI.I, contreplat supérieur et fol. I $r$. C BNCR; consultable en ligne.

À côté de quelques citations (Saba, Novalis, Rimbaud), Morante note de nombreuses propositions de titres pour son roman, publié en 1948. On remarquera au bas du contreplat supérieur le titre «Menzogna e sortilegio », qu'elle souligne trois fois et qui sera finalement retenu. Parmi les multiples choix possibles, figure aussi le titre «La casa dei bugiardi », qui sera utilisé pour la traduction américaine du roman (House of Liars).

Il faut retenir que nous étudions les listes selon leur usage, et non sur la base de leur contenu : une liste de mots isolés peut ainsi être, selon la méthode des écrivains qui l'emploient, tout autant expansive que contractile. Certains écrivains ont recours aux listes de mots isolés dans la phase pré-rédactionnelle, et ce sont les mots eux-mêmes qui déclenchent l'écriture : c'est le cas par exemple pour l'écrivain sicilien Gesualdo Bufalino, qui utilisait ce type de liste comme point de départ à la rédaction.

Il écrivait d'abord une série de lemmes en vrac sur des feuilles volantes, ensuite il les répertoriait selon l'ordre alphabétique et enfin il les mettait les uns à côté des autres selon une méthode qu'il appelait Ikebana, c'est-à-dire un rapprochement qui reposait sur un accord musical ou sur une friction. Une fois ces mots agencés et reliés entre eux, il esquissait une idée dans laquelle il synthétisait une sorte de mélodie, un choix de deux ou trois notes, qu'il juxtaposait à une idée préexistante, laquelle était remaniée et amplifiée par la suite 3 .

$\mathrm{Si}$, chez des écrivains comme Bufalino, les listes de mots isolés peuvent être utilisées en tant que listes expansives, chez d'autres, ces mêmes listes peuvent donner lieu à un mouvement

3. Giulia Cacciatore, «L'Opus Perpetuum di Gesualdo Bufalino» dans Nunzio Zago et Giuseppe Traina (dir.), Il miglior fabbro. Bufalino fra tradizione e sperimentazione, Leonforte, Euno Edizioni, 2014, p. 173-204 : p. 201 (ma traduction). 


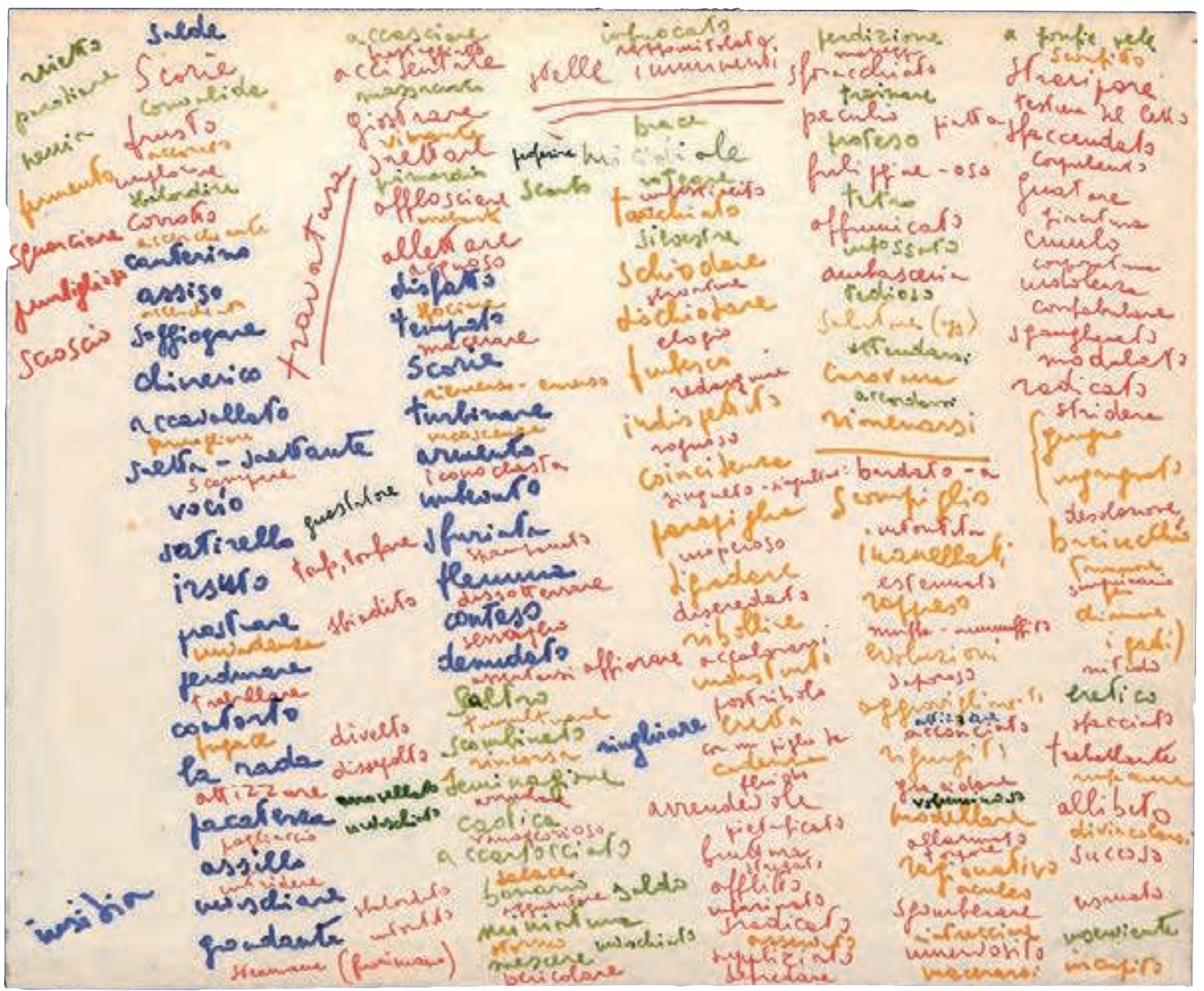

Fig. 2 : Elsa Morante, liste de mots isolés. Dossier de genèse de La Storia.

Rome, Biblioteca Nazionale Centrale, V.E. 1618/1.3, contreplat inférieur. Consultable en ligne.

Dans les dossiers de genèse des romans d'Elsa Morante figurent plusieurs listes de mots isolés. Ces listes peuvent valoir de réservoir d'où Morante puise des expressions tout au long de l'écriture, ou de pense-bête pour les élaborations successives : elles «préparent un lexique de choix, une liste "surveillée" de mots, un répertoire privilégié dans lequel puiser si nécessaire, si bien que les expressions les plus chères à l'auteur sont soulignées » (Simona Cives, «Elsa Morante Senza i conforti della religione», dans G. Zagra, S. Buttò (dir.), Le stanze di Elsa. Dentro la scrittura di Elsa Morante, Rome, Colombo, 2006, p. 57-58).

de contraction, comme les listes lexicales qui, dans la phase de révision, sont utilisées pour éviter des répétitions dans le texte (fig. 2).

Parfois, une liste peut être utilisée en même temps dans la direction de l'expansion et dans celle, opposée, de la contraction : c'est le cas par exemple d'une liste qui figure dans le dossier de genèse de la Proposta di alcune correzioni e giunte al Vocabolario della Crusca de Vincenzo Monti, étudiée par Claudia Bonsi ${ }^{4}$. Pour rédiger sa Proposta, Monti dressa plusieurs listes

4. Claudia Bonsi, «Liste lessicali e nascita di un lessicografo : il caso di Vincenzo Monti », Prassi Ecdotiche della Modernità Letteraria, 3 (2018), sous presse, consultable en ligne. La Proposta, publiée en trois volumes entre 1817 et 1824, avec une annexe parue en 1926, est le résultat d'un ambitieux travail lexicographique que Monti entreprend 
de termes à ajouter au Vocabulaire, souvent organisées selon l'ordre alphabétique, dont une notamment qu'il utilisa pour s'orienter dans la masse d'informations sur lesquelles il était en train de travailler : à la fois expansive (car une partie des items dont elle se compose intégreront, accompagnés de leur définition et d'un commentaire, le texte de la Proposta) et contractile (car elle est également utilisée pour trier les termes à gloser et ceux à laisser tomber), cette liste contient plus que ce qu'il écrira dans la Proposta: la moitié des termes répertoriés sera abandonnée.

Parmi les listes génétiques, les listes expansives sont particulièrement intéressantes, car elles permettent de retracer la genèse d'une œuvre. Dans certains genres littéraires, comme l'autobiographie et le roman historique, ces listes sont un instrument précieux pour saisir les parcours de la création, et le passage des données évènementielles (les événements qui ont marqué la vie de quelqu'un, ou les événements historiques) à leur réécriture fictionnelle. Ainsi, les écrivains qui ont recours aux listes dans l'état préparatoire de leurs mémoires nous donnent l'occasion d'étudier les enjeux du récit autobiographique à l'état naissant : si «le choix des souvenirs et leur montage» sont «deux opérations à la fois nécessaires et relativement invisibles 5 » qui président au discours du narrateur autobiographique, ces deux mouvements apparaissent de façon flagrante dans une liste génétique.

Vittorio Alfieri, par exemple, pour appuyer la rédaction de ses mémoires, dresse des listes de deux types différents : pour les trois premières «Époques 6 » de son autobiographie (consacrées respectivement à son enfance, à son adolescence et à sa toute première jeunesse), il répertorie laconiquement des événements concernant sa formation et ses voyages, alors que pour la quatrième «Époque» (consacrée à sa «Virilité», qu'il fait commencer au moment où il se consacre à la littérature), il répertorie plutôt les travaux littéraires qu'il a accomplis ou entamés. Cette divergence nourrit un écart de tonalité manifeste entre les trois premières «Époques » de Ma vie (où la formation, mais surtout l'aventure et le goût romanesque prennent le dessus) et la quatrième «Époque» qui, portant sur l'itinéraire d'un intellectuel voué à la littérature, se transforme quasiment en un catalogue de travaux littéraires.

La première liste, connue sous le titre éditorial de «prospectus chronologique de $\mathrm{Ma} \mathrm{vie}^{7}$ », tient sur une seule feuille : à côté des années concernées (de 1749 à 1789), Alfieri note les aspects remarquables de sa vie en style télégraphique (fig. 3) :

1781. Voyage à Rome, et Naples, puis je m'installe à Rome.

1782. Les 14 tragédies sont terminées.

1783. Impression de 10 tragédies; voyages et malheurs 8 .

à l'encontre du Vocabulaire de la Crusca, auquel il reproche des manques et des incohérences dans le choix des termes à définir.

5. Philippe Lejeune, Les Brouillons de soi, Paris, Le Seuil, 1998, p. 39.

6. L'autobiographie d'Alfieri (Vita di Vittorio Alfieri da Asti scritta da esso, Florence, Piatti, 1806, [avec une fausse adresse à Londres, 1804], en version française : Ma vie : mémoires écrits par lui-même, traduit de l'italien par Antoine de Latour, Paris, Mercure de France, 2012 [1840]) est structurée en quatre «Époques» («Enfance», «Adolescence», «Jeunesse» et «Virilité»), comportant un nombre inégal de chapitres.

7. Florence, Biblioteca Medicea Laurenziana, manuscrit «Alfieri 13», fol. $230 r$. Ce «prospectus » a été publié par Luigi Fassò dans son édition critique de l'autobiographie d'Alfieri : voir Vittorio Alfieri, Vita, L. Fassò (dir.), Asti, Casa d'Alfieri, 1951, vol. II, p. 275-276.

8. Ibid., ma traduction.

\section{Pouvoir génétique} des listes expansives 


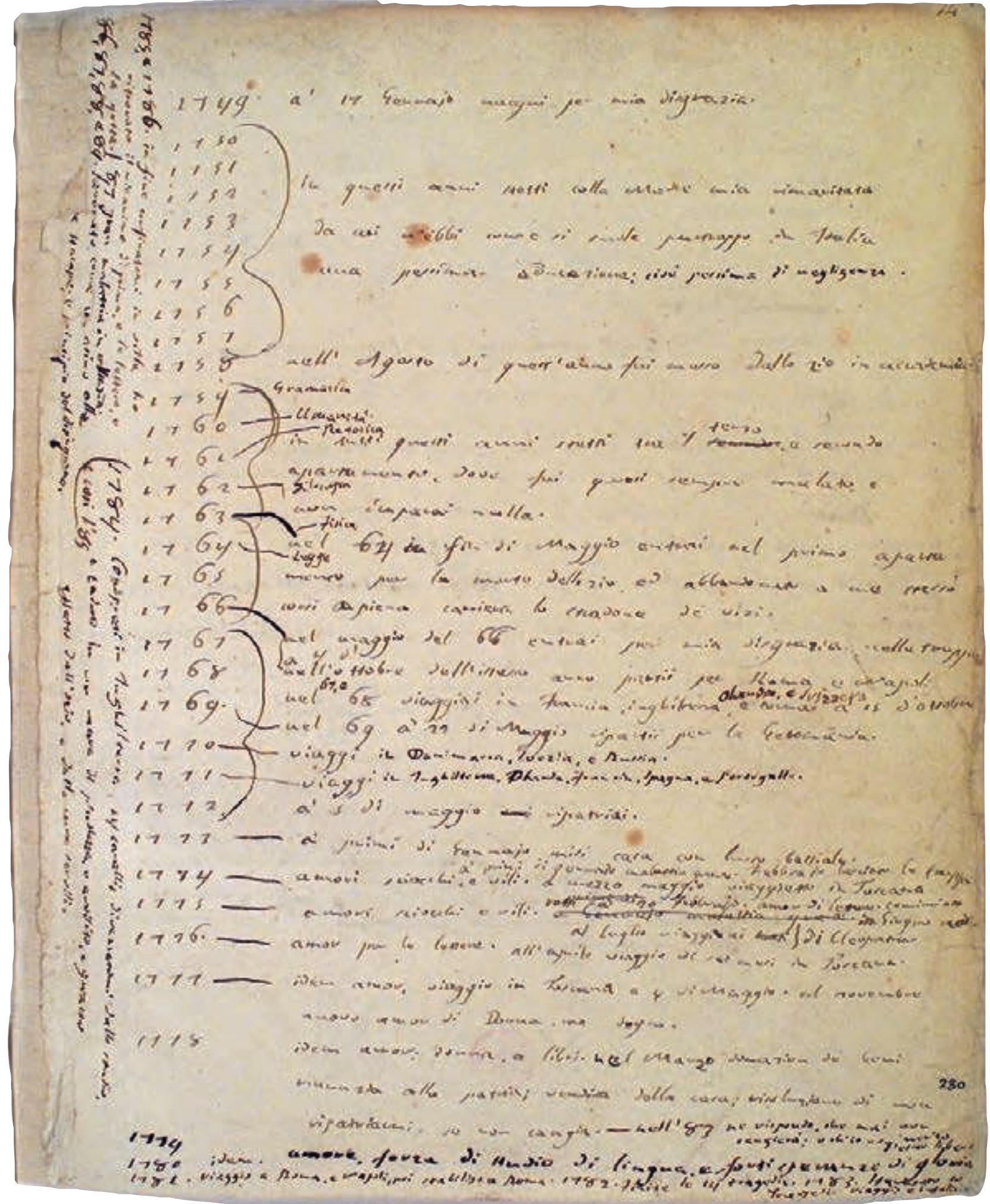

Fig. 3 : Vittorio Alfieri, «prospectus chronologique» pour l'autobiographie (Vita), 1790 ca. Florence, Biblioteca Medicea Laurenziana, ms. «Alfieri 13», fol. 230 r. (c) BML 
Il s'agit, dans ce cas, d'une liste dressée en amont, qu'Alfieri utilisa semble-t-il pour avoir un regard d'ensemble sur son existence jusqu'en 1790 (date à laquelle il entame la rédaction de ses mémoires) et pour concevoir, sur la base de ce survol, la structure de son récit autobiographique. L'aspect extrêmement synthétique de ce document (rigidement sculpté par la succession chronologique) nous met face à la conscience lucide qu'Alfieri avait des faits-clés de son «récit de soi» : l'éducation inadéquate de son enfance, sa formation d'adolescent (toujours inadéquate, selon lui) à l'Académie de Turin, et la grande saison des amours et des voyages à travers l'Europe qui marquent sa jeunesse, jusqu'à la décision de se consacrer entièrement à la carrière littéraire et à la rencontre, enfin, d'un «amour digne ».

Pour ce qui est des événements qui ont marqué la vie de l'auteur, le récit de l'autobiographie d'Alfieri est déjà intégralement esquissé dans ce «prospectus chronologique »; toutefois, bien que tous les éléments y figurent, l'enjeu de ses mémoires semble se nicher parmi les espaces blancs qui séparent les items de cette chronologie. Ma Vie s'organise précisément autour des liens de sens qui tissent cette suite de dates et d'événements, que l'on pense au primat donné à la «conversion » à la littérature, ou aux antinomies et aux parallélismes entre les malheureuses «amours indignes» et le précieux «amour digne» : autant d'éléments qui constitueraient rétrospectivement l'échafaudage d'un abrégé de l'autobiographie d'Alfieri et qui pourtant ne ressortent pas de ce «prospectus chronologique».

Il en va de même pour la deuxième liste préparant son autobiographie, celle consacrée aux travaux littéraires (fig. 4). Intitulée par l'auteur «Reddition de comptes à donner au tribunal d'Apollon ${ }^{9} »$, cette liste fut commencée en 1790 et mise à jour, à intervalles réguliers, entre 1797 et 1803 :

\section{Florence.}

Au mois de mars, versification de la Marie Stuart. En juillet, rédaction de l'Octavie et du Timoléon, et troisième re-versification du Philippe III. Au mois de septembre, versification de la Rosemonde. En décembre, j'ai commencé à mettre en vers l'Octavie. Au cours de l'année, j'ai achevé le deuxième chant du poème et $\mathrm{j}$ 'ai rédigé quelques poèmes ${ }^{10}$.

Cette suite de travaux littéraires (l'intégralité de la «reddition de comptes» suit cette structure) est bien sûr intégrée à la narration autobiographique mais, là encore, elle est absorbée et dépassée par l'agencement du récit : les chapitres qui rythment $M a$ Vie, bien que le récit suive un ordre chronologique - pointé aussi par des datations courantes dans les marges de la page -, s'articulent plutôt autour des événements marquants, dont la mise en scène est soigneusement méditée. En outre, tous les travaux littéraires évoqués par Alfieri dans la «reddition de comptes» sont insérés dans un contexte (aussi bien humain qu'historique) plus vaste, qui dépasse la simple liste des œuvres accomplies, imprimées ou en cours de travail, ce qui leur confère un sens plus profond.

Cette longue liste d'études et de travaux rend bien peu compte de la richesse du récit auquel Alfieri a abouti; elle témoigne néanmoins très nettement de son projet à l'origine : retracer son parcours exemplaire d'écrivain, rythmé par les dates clés de sa formation et des

9. «Rendimento di conti da darsi al tribunal d'Apollo sul buono o mal impiego degli anni virili. Dal 1774 in poi », Florence, Biblioteca Medicea Laurenziana, Manuscrit «Alfieri 10 »; à présent dans Vita (éd. par L. Fassò), op. cit., II, p. 259-271.

10. Ibid., p. 261, ma traduction. 
1774 . Torino.

$$
\text { Anni tetzerayjurists }
$$

Ned Ferbinge,

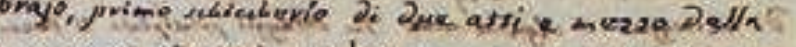

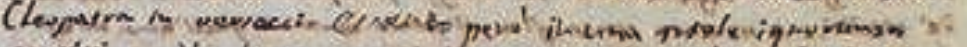

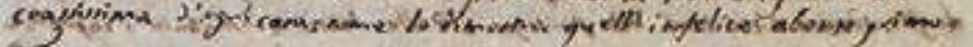

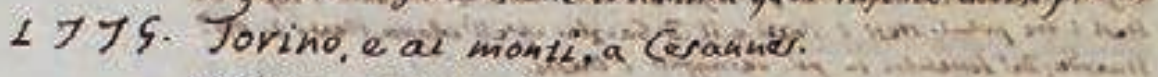

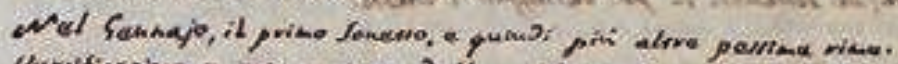

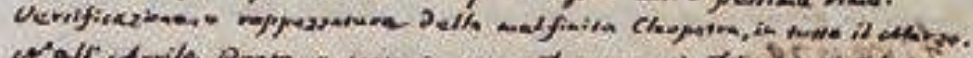

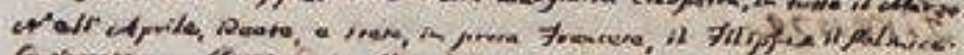

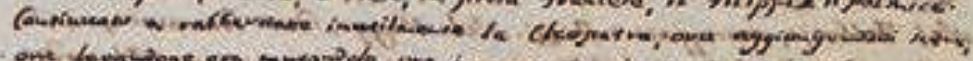

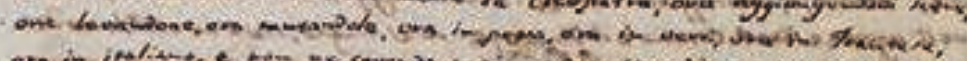

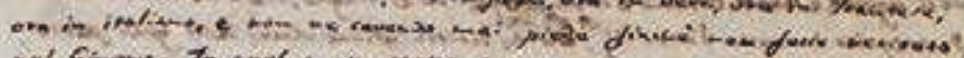

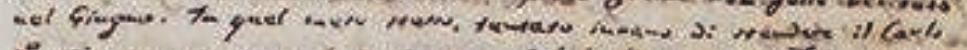

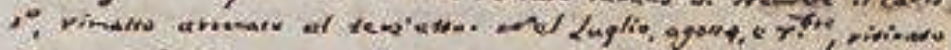

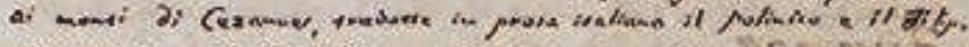

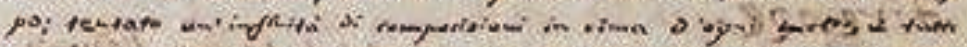

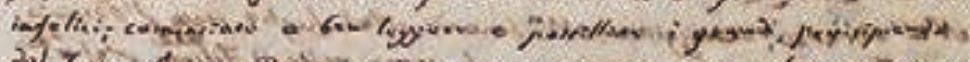

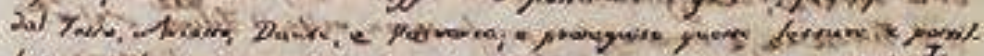

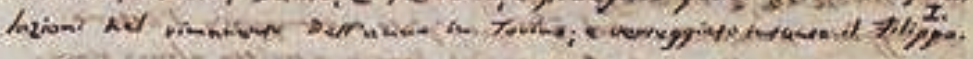

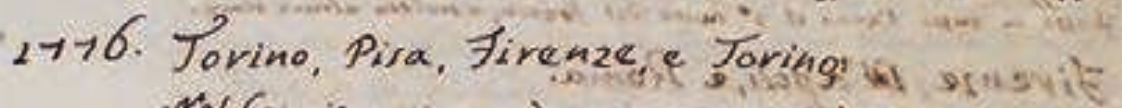

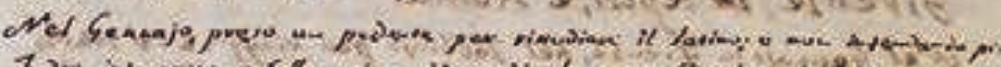

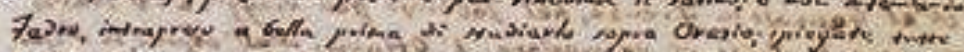

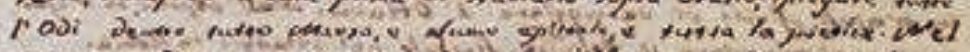

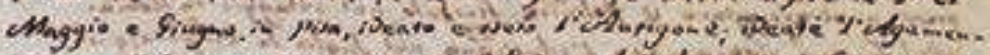

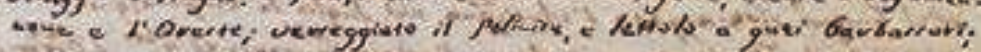

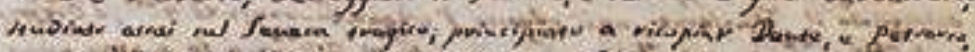

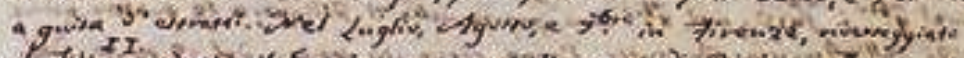

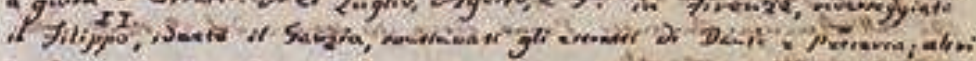

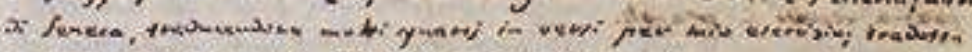

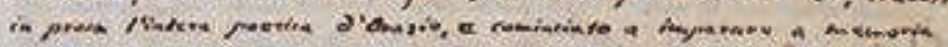

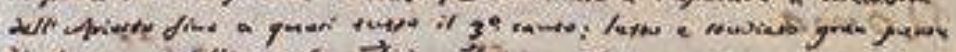

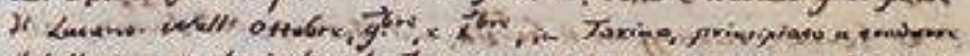

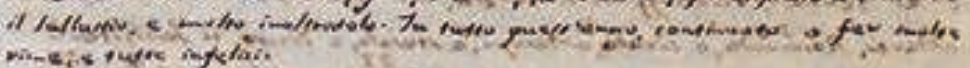

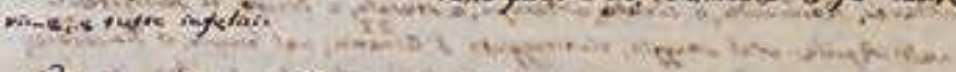

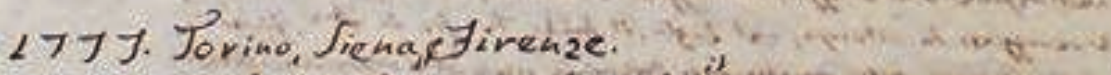

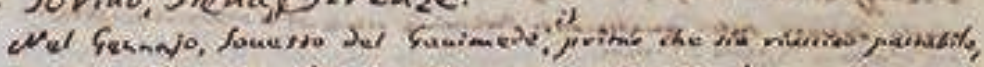

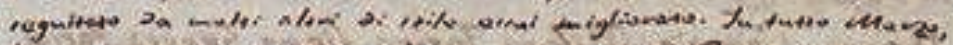

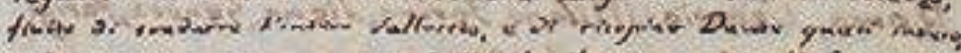

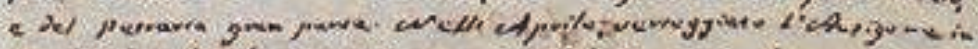

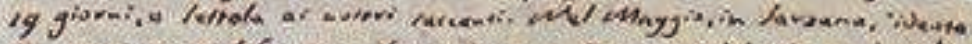

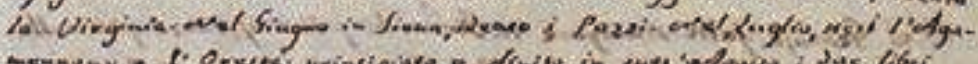

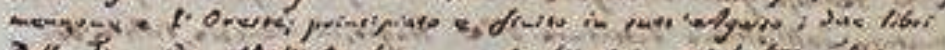

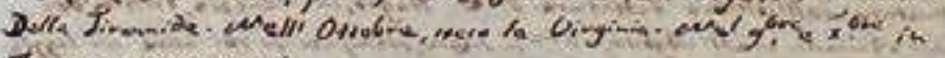
Fiva-2s, vemsomina.

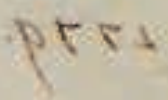

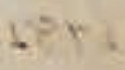


étapes de son œuvre. Si l'on a souvent rapproché Alfieri de Casanova, autre italien voyageur du XVIII siècle qui a légué ses mémoires, c'est une tout autre liste qui préside au récit autobiographique de Casanova. Le dossier de genèse de l'Histoire de ma vie comprend en effet quelques «aide-mémoires » fort précieux pour saisir la construction littéraire de son autobiographie :

Ils se présentent sous forme de listes de noms de personnes, d'anecdotes, de bons mots, de souvenirs dont certains ont été biffés quand ils ont été utilisés ou rejetés. On y voit s'entrelacer notes historiques, références chronologiques, souvenirs personnels, sous forme de juxtaposition de mots ou de phrases rédigées. Si la plupart de ces noms et souvenirs apparaissent dans l'H[istoire de ma] V[ie], d'autres n’y ont pas trouvé leur place ${ }^{11}$.

La très célèbre liste de noms - dont un bon nombre furent biffés au fur et à mesure qu'ils intégraient la narration - montre que les mémoires de Casanova se construisent, d'une façon quasi théâtrale, sur les rencontres qui rythment sa vie privée, ses amours, ses voyages, ses aventures : alors qu'Alfieri déploie sous nos yeux les étapes de la formation d'un écrivain, Casanova retrace la vie d'un libertin. Deux démarches et deux enjeux opposés, que mettent en évidence les avant-textes de ces deux autobiographies : une liste d'ouvrages chez Alfieri, une liste de noms chez Casanova.

Les listes nous permettent également de saisir les enjeux des romans historiques, comme le montre le cas d'Elsa Morante et de son roman La Storia (1974) : dans les avant-textes de ce roman, en effet, figurent de nombreuses listes de sources et de citations, qui permettent de retracer l'intégralité du parcours génétique (des livres lus au livre écrit) : on remarquera que la liste est, très souvent, le dispositif-pivot qui mène de l'exogenèse à l'endogenèse. C'est le cas, notamment, de la liste suivante qui figure dans un petit carnet dont Morante s'est servie pour la réécriture de quelques passages de son roman et où elle rassemble des événements historiques qui ont eu lieu en Calabre après la Première Guerre mondiale (fig. 5). En voici la première partie :

Après-guerre 1918-1922 (notamment en Calabre)

Juin 1919 - famine et faim grèves et désordres publics à La Spezia

Grève générale à Gênes et Pise

Pillages de magasins à Forlì

Révoltes partout surtout à cause de la cherté de la vie

[La pression populaire conduit aux élections de 1919]

En Calabre, les soulèvements concernent surtout les paysans.

En août 1919, la grippe (grippe espagnole?) s'ajoute à la famine. Voir «Le lotte contadine» aux pages $118-119$

1920 - épidémies à Cosenza. Grèves partout en Italie

Diminution forcée de l'émigration (à cause de la démobilisation des industries de guerre des anglo-franco-américains)

N.B. À Reggio C. il y avait surtout des petits propriétaires (pauvres) beaucoup desquels travaillaient aussi comme ouvriers agricoles à Cosenza il y avait surtout des ouvriers agricoles 12 .

11. Gérard Lahouati, «Le long travail», Genesis, n 34, 2012, p. 97-122. Voir notamment la fig. 1 (p. 99).

12. Elsa Morante, Rome, BNCR, fonds Vittorio Emanuele 1618/3.A, fol. $42 r$ et $43 r$, ma traduction. 
Fig. 5. Elsa Morante,

avant-texte de La Storia (19711974). Rome, Biblioteca Nazionale Centrale, V.E. 1618/3.A, fol. $42 r$. (c) BNCR

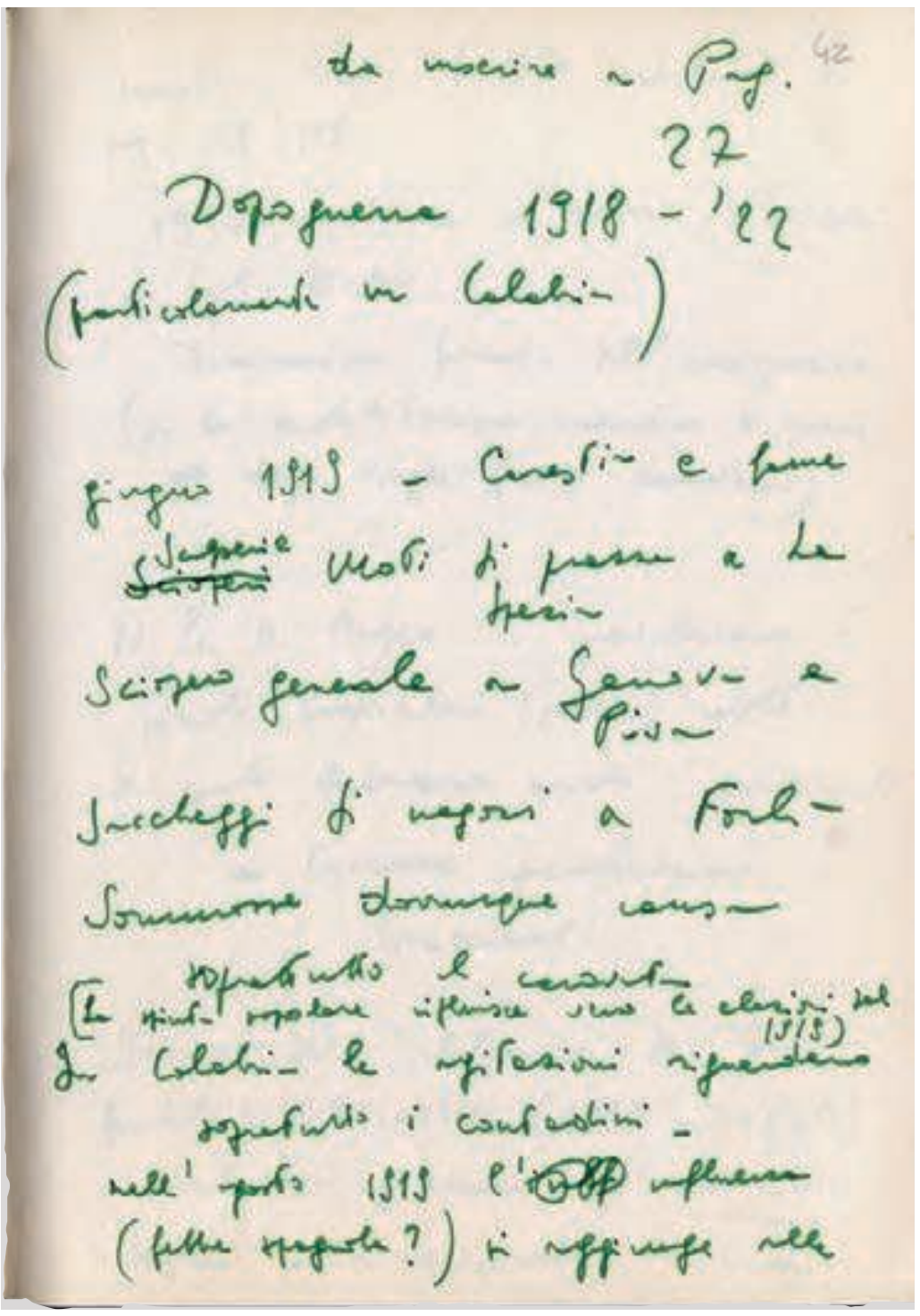

Les items dont cette liste se compose sont à mettre en relation avec des annotations qu'Elsa Morante avait déposées dans les marges d'un volume d'Enzo Misefari consacré aux luttes des paysans, en Calabre, entre 1914 et 192213. L'exemplaire de ce volume qui a appartenu à Elsa Morante, et que j'ai pu consulter ${ }^{14}$, recèle un grand nombre de traces de lecture. Morante transcrit notamment, dans les marges supérieures du livre, un certain nombre d'événements historiques avec leurs dates : «Tribunal de Cosence» (p. 108), «Employés de Cosence» (p. 109), «maîtres d'école de Paola15» (p. 112), «Massacre de Paola» (p. 114), «5 août épidémies» (p. 118), «1920 épidémies à Cosence» (p. 123), «prix en $1921 »($ p. 138), et

13. Enzo Misefari, Le lotte contadine in Calabria nel periodo 1914-1922, Milan, Jaca-Book, 1972.

14. Une grande partie de la bibliothèque personnelle d'Elsa Morante a été léguée à la Biblioteca Nazionale Centrale di Roma (dorénavant : BNCR). Il s'agit d'environ 3900 volumes, qui sont consultables dans la salle «Falqui». L'exemplaire du volume de Misefari ayant appartenu à Morante y figure également : Rome, BNCR, F. MOR., 330 MISEE 1.

15. «Paola» est le nom d'un petit village de Calabre. 
ainsi de suite. Toutes ces annotations sont ensuite utilisées comme pense-bête et recueillies dans la liste chronologique que l'on vient de transcrire. Cette liste sera retravaillée par la suite et constitue le point de départ d'un segment du premier chapitre 16 : la verticalité de la liste se transformera ainsi pour donner naissance à l'horizontalité du récit ${ }^{17}$. Il est clair que nous sommes confrontés, dans ce cas, à une liste dont la fonction est le tri d'informations exogénétiques, au carrefour entre les livres lus et le livre créé. À partir de la narration historique très détaillée du livre de Misefari, l'effort de sélection et de synthèse converge vers la liste, qui, par sa structure, permet à Morante de maîtriser un choix d'informations appelées à être remaniées sous forme narrative, en intégrant à la narration historique (réelle) l'histoire (inventée) des personnages de son roman. Voici ce qui restera de cette liste dans le texte publié (les éléments issus de la liste sont marqués en gras, ce qui concerne les personnages du roman est marqué en italique; d'autres informations tirées du livre de Misefari sont soulignées) :

Les parents de Giuseppe (qui se trouvaient plus bas, dans la province de Reggio) étaient des fermiers qui travaillaient aussi à la journée comme ouvriers agricoles. En août 1919, l'une de ses seurs mourut, ainsi que son mari et deux enfants, de la grippe espagnole. Dans certains pays cette épidémie a laissé un souvenir effrayant. Les médecins, les médicaments et la nourriture faisaient défaut. On était en pleine canicule. Le nombre des morts dépassait celui de la guerre. Et comme il n'y avait pas assez de planches pour les cercueils, les cadavres restaient plusieurs jours sans sépulture.

À cette époque, Giuseppe envoyait à ses parents tout son traitement (qui, étant donné les actuelles difficultés publiques, ne lui était pas toujours payé régulièrement). Et, malgré la cherté de la vie, les trois Ramundo devaient se débrouiller avec le seul traitement de Nora18.

Les faits historiques, et notamment ceux qui étaient regroupés dans la liste qu'on vient d'examiner, sont portés du niveau impersonnel et général de la narration historique de Misefari au niveau de l'histoire particulière des protagonistes du roman : autour des items de cette liste chronologique, Morante tresse ainsi le récit de La Storia dont l'objectif, comme elle le déclara par la suite, était précisément de laisser un témoignage de l'expérience terrible que fut la Seconde Guerre mondiale. Plusieurs passages de La Storia ne sont pas simplement inspirés de, mais effectivement empruntés à d'autres ouvrages : il est toutefois intéressant d'observer que Morante ne fait appel aux listes que dans le cas des sources historiques. Cette liste nous permet ainsi de percevoir un aspect très important de la poétique de La Storia : les événements historiques constituent pour Elsa Morante une simple grille au sein de laquelle

16. Elsa Morante, La Storia, traduit de l'italien par Michel Arnaud, Paris, Gallimard, 2003 (19771; en langue originale : Turin, Einaudi, 1974), p. 49-50.

17. Par «horizontalité du récit», je n'entends pas un récit conservant la forme de l'énumération, comme c'est le cas chez Marguerite Yourcenar. Lucia Manea, en étudiant le dossier de genèse de L'Euvre au Noir, riche en listes, fait en effet l'observation suivante : «Si la liste est associée à la verticalité, une fois insérée dans le récit elle s'appuie sur une pratique rhétorique (accumulation, dénombrement, énumération), emprunte la forme d'une série déployée horizontalement. L'énumération transpose et métamorphose ainsi le contenu des listes de l'avant-texte» (Lucia Manea, «L'effet générateur de fiction de la liste. Variations - des carnets de composition au roman », dans S. Milcent-Lawson, M. Lecolle et R. Michel (dir.), Liste et effet liste en littérature, Paris, Classiques Garnier, 2013, p. 97-109 : p. 101). Dans le cas de Morante, en revanche, le récit s'affranchit de la liste qui l'a engendré.

18. Morante, La Storia, op. cit., p. 51. 
elle va insérer, comme en autant de cases, la fiction (notamment, l'histoire inventée des personnages du roman), et la création littéraire se développe dans les replis des espaces blancs qui séparent les items de cette liste. À travers l'usage qu'elle fait de cette liste, Elsa Morante suggère que l'Histoire n'est qu'une liste de dates et d'événements, et que la vie et l'art demeurent ailleurs, dans les liens narratifs qui agencent et harmonisent les items bruts d'une chronologie, en leur attribuant un sens et en les absorbant dans un discours articulé, susceptible de dépasser le cadre étroit de la liste et de transformer en poésie son enchaînement rudimentaire.

\section{Listes de régie et listes d'appui}

Si les listes expansives montrent le processus par lequel le texte s'affranchit de la liste qui l'a engendré, dans d'autres cas les listes sont utilisées pour organiser et structurer la matière littéraire : les items dont elles se composent sont autant de références synthétiques à des blocs de texte que la liste ordonne et agence, ou ré-agence. On parlera alors de listes de régie. La fonction génétique de telles listes repose expressément sur leur structure : c'est l'ordre des items, leur organisation (ou réorganisation), qui travaille le devenir d'une œuvre. Tout comme les listes expansives et contractiles, les listes de régie sont appelées à disparaître au fil de l'écriture, bien que le texte ou l'œuvre doivent garder - du moins en partie - la mémoire de la structure de la liste génétique qui les a nourris. Elles sont utilisées pour organiser les éléments d'un texte en préparation et les déplacer comme des blocs modulaires, afin de dessiner ou redéfinir l'architecture d'ensemble. Il s'agit de listes qui ressemblent à des tables des matières (et qui, parfois, en feront office) : bien qu'elles ne président pas strictement à la naissance de l'écriture, les listes de régie (qui l'organisent, dans la plupart des cas, rétrospectivement) ont tout de même à voir avec la naissance d'une œuvre. Les items de la liste constituent, dans ce cas, le référentiel synthétique des différentes parties d'un texte (chapitres, épisodes) ou d'un recueil (poèmes, contes). Andrea Zanzotto en faisait notamment un usage extensif. Dans le souci d'organiser ses poèmes au sein d'un ensemble cohérent, il dressait plusieurs tables des matières, afin de redistribuer les poèmes selon une architecture soigneusement soupesée : le macro-texte redessine en effet un parcours idéal à travers la répartition des poèmes dans trois recueils (Le Galatée au bois, Phosphènes et Idiome) qui donne lieu à une trilogie unitaire, dont les interrelations (ainsi que la longue gestation) sont attestées par les nombreux plans rédigés par Zanzotto ${ }^{19}$.

Parfois, les listes de régie témoignent en même temps de la préparation d'un texte et de sa réorganisation. Que l'on pense par exemple aux papiers préparatoires de l'Éloge d'Esprit Fléchier par D'Alembert, où figure

une liste de points à aborder dans le texte, dans un ordre qui n'est pas celui du texte imprimé, ce qui confère au document moins le statut de plan que celui de réservoir de données, dans une phase précoce du processus d'invention. C'est ce que suggère l'ajout de numéros, modifiables par la suite, qui seraient la trace du travail de disposition. C'est aussi ce que suggère le fait que tous les développements ont été biffés, la biffure témoignant de l'accomplissement, ailleurs et ultérieurement, de la rédaction de chaque développement présent ici à l'état embryonnaire : confrontant son texte

19. Voir Genesi e storia della «trilogia», op. cit., notamment les p. 167-183. 
avec les éléments contenus dans le document, D'Alembert vérifierait en somme qu'il n'a rien oublié de son programme initial 20 .

Il y a, enfin, l'ensemble très varié des listes d'appui, dont le rôle dans la genèse des œuvres demeure plus insaisissable, car elles ont normalement une fonction auxiliaire et ne nourrissent pas directement la rédaction d'un texte. Dans la plupart des cas, elles sont en effet utilisées comme réservoir ou comme témoignage : bien qu'elles exposent les sources ou les enjeux d'un ouvrage, elles ne participent pas directement de sa genèse. Des listes de mots isolés, par exemple, peuvent être utilisées comme listes d'appui, notamment dans le cas où elles sont utilisées «entre deux langues» : on trouvera ainsi chez les traducteurs des listes de lemmes ou d'expressions traduites ${ }^{21}$, tout comme des glossaires personnalisés sont fréquemment rédigés dans le cadre de l'apprentissage d'une langue. Cette fonction d'appui peut, dans ce type de cas, avoir vocation à emprunter, du moins en partie, le chemin de l'expansion puisque le traducteur construira ses phrases autour d'un choix lexical donné (et réfléchi au préalable). Que l'on pense à Donaldo Schüler, qui a traduit en portugais Finnegans Wake de James Joyce : en étudiant ses carnets de traducteur, Marie-Hélène Paret-Passos décrit un cahier qui «commence par une liste de fragments de phrases, en portugais, suivie de mots, toujours en portugais, accompagnés d'une définition. Ce sont des mots archaïques, inusités, ou des régionalismes ». Pour la critique, cette quête lexicale «atteste qu'une des premières étapes, sinon la première, du travail de recherche a porté sur l'organisation des outils lexicaux, probablement dans le but de se constituer un vivier de mots singuliers, totalement absents du quotidien. D'entrée de jeu, il est possible d'entrevoir le projet qui se dessine, de rester dans le rythme joycien ${ }^{22}$.» Même l'écrivain de «langue seconde» a recours aux listes pour se constituer peu à peu un vocabulaire de choix, destiné à figurer souvent dans ses œuvres. C'est le cas par exemple du Piémontais Vittorio Alfieri (1749-1803) qui, en tant que francophone, pour apprendre la langue italienne rédigeait des listes de mots en italien avec leur traduction en français et, parfois, aussi en dialecte piémontais (fig. 6) 23 .

Néanmoins, lorsqu'elles ont une fonction d'appui, il est rare que les listes participent directement de la genèse d'un ouvrage. Parmi les listes d'appui on peut mentionner les listes bibliographiques, que certains écrivains dressent pour témoigner de leurs choix lexicaux ou pour garder la trace des sources qui ont nourri leur création. Dans le dossier de genèse du roman La Storia (1974), par exemple, figurent des listes de références bibliographiques, qu'Elsa Morante a rédigées au cours de la phase de relecture du roman. Ces listes, autrement dit, n'apprivoisent pas l'écriture et ont très souvent une fonction de témoignage, comme si elles n'avaient été rédigées qu'à l'usage de la postérité. C'est le cas, entre autres, d'une liste de sources iconographiques que l'auteure dresse à côté d'un passage de La Storia où le petit

20. Olivier Ferret, Alexandre Guilbaud et Irène Passeron, «Pleins et déliés dans les manuscrits de D’Alembert», Genesis, $\mathrm{n}^{\circ} 34,2012$, p. 67-82.

21. Voir, dans ce même numéro, l'exemple de la traduction du poème I limoni d'Eugenio Montale par Gustav Roud, porté par Rudolf Mahrer (p. 47, fig. 5). Après avoir ébauché une traduction du poème, Roud note dans les marges des termes ou expressions en italien qu'il se propose de «chercher» dans le vocabulaire. Quelques expressions restent sans traduction (voir par exemple «mettere ici :» ou encore « talora :»).

22. Marie-Hélène Paret-Passos, «De Finnegans Wake à Finnicius Revém», Genesis, n 38, 2014, p. 69-84 : p. 79.

23. Voir Gian Luigi Beccaria, «Prefazione» dans Vittorio Alfieri, Appunti di lingua e letterari. Con un'appendice di aggiunte ai volumi pubblicati, G.L. Beccaria et M. Sterpos (dir.), Asti, Casa d'Alfieri, 1983, p. 9-26 : 14-19. 


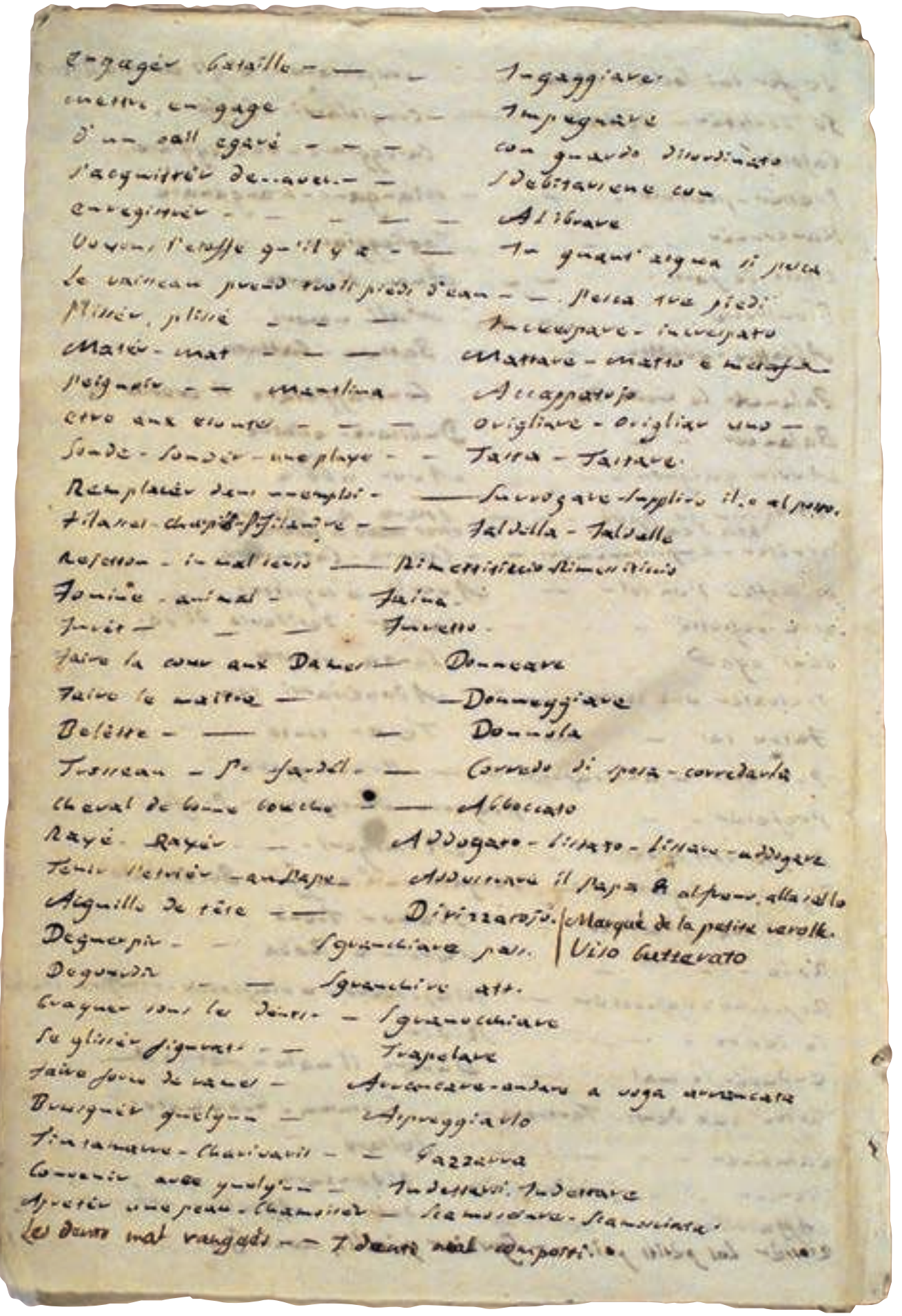

Fig. 6. Vittorio Alfieri, liste d'expressions en français avec leur traduction en italien, 1778.

Florence, Biblioteca Medicea Laurenziana, ms. «Alfieri $10 »$, fol. $13 v$. () BML

Né dans le Piémont et francophone, Alfieri ne se décida à devenir un écrivain de langue italienne qu'à partir de 1775 seulement : il engagea ainsi un véritable combat grammatical et linguistique pour parvenir à la maîtrise de la langue toscane. Voir Ch. Del Vento et G. Santato (dir.), Quand Alfieri écrivait en français. Vittorio Alfieri et la culture française, Cuneo, L'artistica Savigliano, 2003, fiche n ${ }^{\circ} 9$ «Alfieri écrivain entre français et italien. Les Appunti di lingua», p. 47-48. 
protagoniste du roman, Useppe, un enfant de moins de cinq ans, est confronté à des images de guerre (fig. 7) :

Pour les photos citées, voir :

1) Amas de cadavres

1) Partisans pendus (voir épisode précédent album Incenso e polvere Prunas pag.

2) Mussolini pendu (épisode précédent) - id.

3) Jeune fille collaborationniste (épisode précédent) Album Robert Kapa - Images of War pag. 134

4) Amas de cadavres (The trial of Adolf Eichmann - pag. 229)

5) Tas de souliers (v. The History of the Gestapo)

6) Escalier de la mort (v. Tu passerai per il camino)

7) Prisonniers des camps de concentration (souvenir à retrouver)

8) Expériences en chambre de décompression (souvenir à retrouver) $\downarrow$

Voir, pour quelque chose de comparable, The History of the Gestapo photographie $\mathrm{g}^{24}$

Dans le roman, ces images sont présentées et décrites du point de vue d'un enfant qui voit ces choses sans en comprendre le sens : «il restera à jamais impossible de savoir ce que ce pauvre analphabète d'Useppe a bien pu comprendre dans ces photos dénuées de sens pour lui. 25 » Si ces listes d'appui sont d'une grande aide pour le commentateur (dans ce cas, elles nous permettent d'appréhender la transmutation de l'image en texte, tout en retraçant la transformation de la source historique dans le cadre de sa réécriture fictionnelle), il convient de se demander pourquoi Elsa Morante les a rédigées alors qu'elles ne sont pas destinées par la suite à figurer dans son roman, et n'occasionneront pas une réécriture du passage concerné. Cette pratique a évidemment trait aux enjeux de La Storia qui, pour Elsa Morante, avait presque la valeur d'un compte rendu de la terrible expérience collective qu'avait représentée la Seconde Guerre mondiale. Ces listes de sources confirment son souci de véridicité et son attention à la représentation exacte de chaque élément, même au détail le plus anodin en apparence. D'autre part, ces listes lui servaient probablement d'aide-mémoire et de réserve d'informations lors de la révision et de la vérification de son texte.

Nous avons cherché, à travers cet éventail de listes génétiques, à lancer des pistes de réflexion sur le pouvoir génétique des listes qui ne sont pas destinées à figurer sous forme de liste dans le texte publié, tout en cherchant à en tracer les chemins génétiques : comme nous l'avons précisé au début de cette réflexion, notre analyse portait sur les listes dressées sans souci de rhétorique, destinées à être retravaillées au fil de l'écriture, jusqu'à leur disparition. La liste, qu'elle soit le point d'arrivée de l'écriture ou bien son point de départ, qu'elle soit destinée à être absorbée par l'agencement du récit ou bien à s'émanciper progressivement d'un texte agencé, se révèle un dispositif précieux pour le généticien : elle permet de préciser la méthode de travail d'un auteur, tout en offrant un angle d'attaque privilégié pour saisir les enjeux des ouvrages étudiés ; mais c'est surtout le rapport entre liste et texte agencé qui nous semble faire ressortir le pouvoir de ce dispositif : les espaces blancs qui séparent les items

24. Elsa Morante, Rome, BNCR, fonds Vittorio Emanuele 1618/1.X, fol. 28 v, ma traduction.

25. Elsa Morante, La Storia, op. cit., p. 534. 


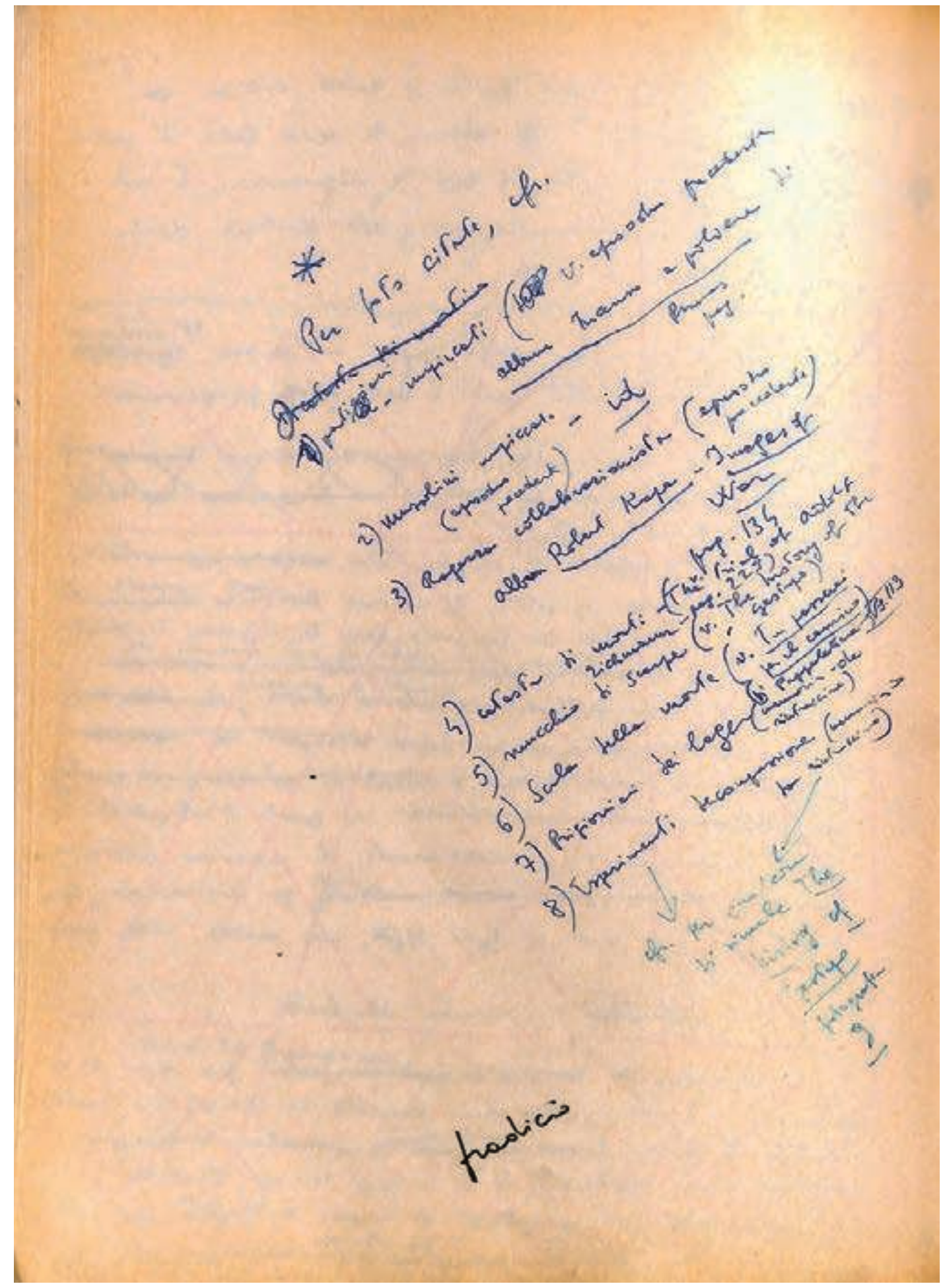

Fig. 7. Elsa Morante, avant-texte de La Storia, cahier X (1971-1972).

Rome, Biblioteca Nazionale Centrale, V.E. 1618/1.X, fol. $28 v$. ( B BNCR; consultable en ligne.

Plusieurs listes bibliographiques figurent dans le dossier génétique de La Storia : elles permettent de retracer le passage de l'exo- à l'endogenèse et mettent en exergue un aspect très important de la poétique de ce roman : l'Histoire n'est qu'une liste stérile de dates et de faits, alors que la richesse de la vie demeure dans le geste artistique de l'écriture, qui agence ces éléments dans le cadre de la fictio narrative. 
d'une liste interrogent la virtualité des possibles, et c'est dans ce combat entre condensation et expansion que l'on retrace les chemins génétiques d'un ouvrage. S'accompagnant, selon les cas, de l'ajout ou de la suppression des items, la fonction des listes dans la genèse littéraire semble ainsi reposer avant tout sur deux mouvements opposés de dilution et de condensation, qui questionnent le rôle des espaces blancs : face à un mouvement d'expansion, la dilution des items sera contrebalancée par la création de matière supplémentaire (les noirs de l'écriture remplissant au fur et à mesure les espaces blancs d'une liste) alors que, dans une démarche de condensation, la genèse reposera sur la création de ces mêmes blancs. 
MONICA ZANARDO est membre de l'équipe «Écritures des Lumières» de l'ITEM, où elle participe aux activités du groupe de travail Manuscrits italiens. Ses recherches portent sur les archives littéraires italiennes et sur les bibliothèques d'écrivains (XVIII ${ }^{\mathrm{e}}$-XXe siècles). Elle est auteure de l'essai Il poeta e la grazia. Una lettura dei manoscritti della Storia di Elsa Morante (Rome, Edizioni di Storia e Letteratura, 2017) consacré au dossier de genèse du roman La Storia d'Elsa Morante; elle prépare l'édition génétique numérique des archives littéraires de Vittorio Alfieri.

monicazanardo@gmail.com

\section{Avant l'écriture, à côté du texte : listes et genèse littéraire}

L'article porte sur les listes génétiques qui figurent dans les papiers autographes de plusieurs écrivains et qui sont destinées à disparaître au fil de l'écriture. Nous proposons un classement fonctionnel des listes génétiques sur la base de leurs usages : aux listes «expansives» (qui sont progressivement développées au fil de l'écriture) s'opposent les listes «contractiles» (sur lesquelles les écrivains travaillent par sélection progressive des items). À côté de ces deux directions de dépassement de la liste, on trouve aussi les listes d'appui (qui se situent généralement «à côté du texte», car elles ne participent pas directement de sa genèse) et les listes de régie, visant à réorganiser la matière littéraire.

This paper focuses on "genetic" lists which may be found in the manuscripts of several writers, and which have usually disappeared in the final version of the printed text. The aim is to classify such lists based on their functions, ranging from "expansive" lists (which are likely to be progressively developed) to "contractive" lists (in which writers work through a gradual selection of items). Alongside these two typologies, we find what we could be labelled "service" lists (which are usually "in the margin" of the text, as they are not directly involved in its genesis), as well as "structuring" lists, used for the disposition of materials and contents.

Der Artikel beschäftigt sich mit den genetischen Listen, die in den Handschriften mehrerer Autoren erscheinen und im Laufe des Schreibens verschwinden werden. Wir schlagen eine funktionelle Klassifizierung der genetischen Listen auf der Grundlage ihrer Verwendungen vor: den „expansiven“ Listen (die im Laufe des Schreibens schrittweise entwickelt werden) stehen „kontraktive“ Listen gegenüber (aus denen die Autoren nach und nach Punkte auswählen). Neben diesen beiden Richtungen der Auflösung der Liste gibt es auch die Hilfslisten (die in der Regel ,neben dem Text" stehen, weil sie nicht direkt an seiner Entstehung beteiligt sind) und die Regie-Listen, die darauf abzielen das literarische Material zu reorganisieren.
Este artículo analiza las listas genéticas que figuran en los papeles autógrafos de numerosos escritores y que están destinadas a desaparecer en el proceso de escritura. Proponemos una clasificación funcional de las listas genéticas basada en sus utilizaciones: a la listas "expansivas" (que se desarrollan progresivamente a lo largo de la escritura) se oponen las listas "contráctiles" (que los escritores elaboran seleccionando progresivamente los ítems). Paralelamente a estas dos direcciones de superación de la lista, encontramos también listas de apoyo (que se sitúan por lo general "al margen del texto", puesto que no participan directamente en su génesis), así como listas de armado, destinadas a reorganizar el material literario.

Este artigo ocupa-se de listas genéticas que figuram nos autógrafos de certos escritores e que são destinadas a desaparecer no decurso da escrita. Com base no seu modo de emprego, propõe-se uma classificação funcional dessas listas genéticas: às listas expansivas (que se vão gradualmente desenvolvendo ao correr da escrita) opõem-se as listas contractas (em que os escritores operam uma seleção progressiva de itens). A par destes dois sentidos de evolução da lista, há também listas de apoio (que se situam geralmente "ao lado do texto", sem participar diretamente na sua génese) e listas directivas, que visam reorganizar o material literário.

I 1 contributo è dedicato alle liste genetiche che si trovano nelle carte autografe di numerosi scrittori e che sono destinate a essere superate nel corso delle molteplici riscritture. Proponiamo una classificazione funzionale delle liste genetiche sulla base del loro utilizzo: alle liste "espansive" (che sono progressivamente sviluppate in corso di scrittura) si contrappongono le liste "contrattili" (sulle quali gli scrittori lavorano per selezione progressiva degli elementi). Accanto a queste due direzioni di superamento della lista, si trovano, inoltre, le liste di servizio (che si collocano in genere "accanto" al testo, dal momento che non partecipano direttamente alla sua genesi) e le liste di regia, usate per organizzare il materiale letterario. 